\title{
THE MODERN ROLE OF VISUAL OBSERVATIONS OF COMETS
}

\author{
S.M. LARSON \\ Lunar and Planetary \\ Laboratory \\ University of Arizona \\ Tucson, Arizona 85721 \\ USA
}

\author{
S.J. EDBERG \\ Jet Propulsion Laboratory \\ California Institute of \\ Technology \\ Pasadena, California 91109 \\ USA
}

\author{
D.H. LEVY \\ Lunar and Planetary \\ Laboratory \\ University of Arizona \\ Tucson, Arizona 85721 \\ $U S A$
}

\begin{abstract}
This paper reviews the role of past and future visual observations in cometary research. The strengths and limitations of visual observations are explored for the benefit of both investigators who might have to use them and observers who wish to make real contributions to the field. We consider the characteristics of the eye-brain combination as a detector and compare them with those of modern detectors. We specifically evaluate visual discoveries, magnitude estimates, and drawings.
\end{abstract}

\section{Introduction}

Modern remote sensing of comets relies upon objective electronic and photographic detectors to quantify the comets' reflected and emitted radiation. There are, however, a few situations where contemporary or historical visual observations are used in the study of comets. We identify three main areas where visual observations may contribute to cometary studies:

(1) Discoveries resulting from visual searches.

(2) Monitoring of general cometary activity through visual brightness estimates.

(3) Study of reliable, mostly pre-photographic era, observations of coma morphology.

Although most investigators today prefer the objective and quantitative data obtained with electronic detectors, there are some situations when the eye is the most convenient detector or the only detector available. Interpretation of visual observations requires consideration of the response characteristics and interpretive abilities of the eye and brain in correlating their observations with other types of observations. We review the advantages and limitations of visual observations and cite examples of their use in modern cometary research. Although we try to refrain from making distinctions between professional and amateur astronomers, it should be noted that visual observations from the 19th century were made mostly by professionals, while recent visual observations are made almost exclusively by amateurs. We find that the primary advantage of current visual observations lies in the fact that many observers with moderate-aperture telescopes and with a wide geographical distribution are able to observe when the major observatories are clouded out or are devoted to other pursuits. 
It is beyond the scope of this paper to discuss all of the details of visual observing techniques and analysis of the data or provide new visual data results; these are usually well covered in the cited references. We also note that with larger telescopes and better photographic and electronic detectors, amateur astronomers continue to make useful non-visual contributions to cometary studies. Although the potential role of these non-visual techniques in obtaining astrometric, morphologic, and photometric data by amateurs will continue to grow, a discussion of these techniques is beyond the intent of this paper.

\section{Historical Perspective}

There is a rich historical tradition of visual observations of comets, starting with the era when the eye was the only detector. It was during this time that cometary phenomena were first being characterized, and many of the fundamental concepts of cometary processes were first identified.

\subsection{DISCOVERIES}

Documented naked eye discoveries go back as far as 1095 B.C. (Ho Peng Yoke, 1962), and the Chinese alone recorded a mean rate of 20 comet apparitions per century (Kresak, 1982). There are accounts of multiple naked-eye comet discoveries in medieval times. Toscanelli, who drafted Columbus' map, apparently found 1457 I and possibly 1457 II and 1472 (Vsekhsvyatskii, 1958).

Gottfried Kirsch made the first telescopic comet discovery in November 1680 (Kronk, 1984), but this was an accident, as he was observing the Moon and Mars when he first saw the comet. It appears that Charles Messier was the first to discover comets as part of a systematic program. Since his first discovery took place on January 26, 1760 (Marsden, 1986), one can speculate that his program was inspired by his observations of Comet Halley in 1758 and 1759. In any event, Messier is credited with discovering 12 comets, and he developed the concept of comet hunting as an organized activity. He also compiled his famous catalog of nonstellar objects. In England, Caroline Herschel discovered her first of eight comets, in 1786. The prolific French comet discoverer Jean Louis Pons discovered the first of 37 comets, in 1801. In the United States, E. E. Barnard discovered 16 comets, the last of which (P/Barnard 3 in 1892) was also the first comet to be found photographically. More recently, visual comet discoveries are being made mostly in Japan, Australia, and the United States. We should note that multiple visual discoveries are being made by only a handful of observers carrying out persistent systematic surveys.

\subsection{MAGNITUDE ESTIMATES}

Visual magnitude estimates go back many years and, after astrometry, provide the longest baseline of comet data available (Green and Morris, 1987). It is this extensive database that argues for continued visual brightness estimates. The total visual brightness of a comet is, in some way, a measure of the comet's activity, and brightness curves have been constructed to characterize this activity as a function of heliocentric distance. This is usually done in terms of an absolute total magnitude and a heliocentric brightness variation exponent $\mathrm{n}$ (for example, see Roemer, 1976, and Meisel and Morris, 1976). In some wellobserved comets, both variables may change over different segments of their orbits. Studies of cometary brightness behavior and lists of the standard brightness parameters can be 
found in Holetschek (1896-1917), Bobrovnikoff (1941), Meisel and Morris (1976), and more recently a continuing series of papers by R. Bouma in the Australian journal Perihelion (edited by D. Seargent). For comets well-observed over ranges of geocentric and heliocentric distances, these brightness parameters may be useful for predicting the brightness of those comets in future apparitions. Most of the total visual magnitude estimates have been compiled into the archive of the International Comet Quarterly (ICQ) (Green et al., 1986). With nearly 30,000 entries, most of which are visual (Green, private communication, 1989), it is the largest and most comprehensive database of such observations.

\subsection{COMA MORPHOLOGY}

Pre-photography visual drawings from the past provide information on the rare "great" comets that often showed much changing detail in their dust comae. A classic example is the work of F.W. Bessel, who, in 1835, observed material being ejected towards the Sun from Comet P/Halley and formulated the concept of the "fountain" model of dust ejection from a solid nucleus. G.P. Bond's drawings of Comet Donati in 1858 showed expanding envelopes consistent with the fountain model. Other drawings of P/Halley from the 1835-36 apparition, by H. Schwabe, F.G.W. Struve, J.F.W. Herschel, and T. Maclear (see Rahe et al., 1969, and Donn et al., 1986), show many of the jets and envelope morphology that even today may provide constraints on the nucleus spin vector for that epoch.

\section{The Eye as a Detector}

As for any astronomical observation, correct interpretation requires an understanding of the photometric response of the detector and any reduction or translational biases. The eye-brain combination is a remarkable sensing system that varies among individuals according to age and other factors, and, like other senses, can be trained to respond significantly better than the average. Such training, usually obtained through experience at the telescope, can substantially improve the individual's perception of faint objects or of small features glimpsed during a moment of good seeing. Visual perception, affected in varying degrees by the physical and emotional state of the observer, is an inherently subjective process subject to statistical probabilities. As such, it may be difficult to establish uncertainties for a particular observation. Out of necessity, astronomers from the pre-photography era were more highly trained to see and record details seen through the eyepiece. Today, there are many amateur astronomers with highly developed observing skills. The general characteristics of the eye as described below refer to a "normal" eye, and it should be understood that there may be significant variations. In the remainder of this review, "eye" refers to the "eye-brain combination."

\subsection{SPECTRAL SENSITIVITY}

Through natural selection, the spectral sensitivity of the eye in daylight (photopic) peaks near the solar intensity maximum at $555 \mathrm{~nm}$ and drops to the $10 \%$ level at $470 \mathrm{~nm}$ and $650 \mathrm{~nm}$. The spectral sensitivity peak of the dark adapted (scotopic) eye is shifted to $510 \mathrm{~nm}$, with $10 \%$ levels at $420 \mathrm{~nm}$ and $580 \mathrm{~nm}$ (called the Purkinje shift), and is therefore most representative of the observer's eye response. Comparing this with the spectrum of a "typical" comet (Fig. 1), it is clear that the eye is seeing a combination of reflected solar 


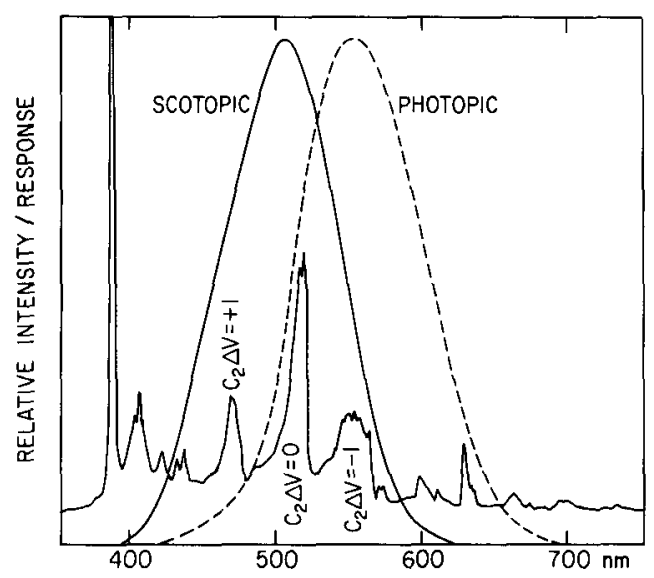

Fig. 1. The spectral sensitivity of the photopic and scotopic eye compared with the emissions of a "typical" comet (in this case, P/Tuttle at $r=1.2 \mathrm{AU}$ ). The relative contributions of the diatomic carbon and reflected solar continuum will vary according to the dustto-gas ratio of the comet and its heliocentric distance.

continuum from dust and major emissions from the $(0,0),(1,0)$, and $(0,1) \mathrm{C}_{2}$ Swan bands. Contributions from $\mathrm{NH}_{2}, \mathrm{Na}$, and $\mathrm{CO}^{+}$may also be seen, but will be minor compared with $\mathrm{C}_{2}$ and continuum. Because of the difference in apparent scale lengths of the dust and gas, a predominantly gaseous comet will appear visually less condensed than a predominantly dusty one. When a comet's heliocentric distance is greater than about $2.5 \mathrm{AU}$, the $\mathrm{C}_{2}$ emission is usually very weak, or not present, so the visual observer is seeing reflected solar continuum. For well-observed, dust-poor comets, it should not be surprising to see an increase in the rate of pre-perihelion brightening at the onset of the $\mathrm{C}_{2}$ emission.

\subsection{DYNAMIC RANGE}

The eye is sensitive to a wide range of intensities, due, in a minor way, to the mechanical action of the iris in bright light and, more importantly, to photosensitive pigments in the rods and cones of the retina. The regeneration of the rod pigment rhodopsin in the rhodopsin cycle increases the sensitivity of the rods after being bleached out by bright light. Most eyes will change their threshold sensitivity by some four orders of magnitude over 30 minutes of dark adaptation. The rate of change is governed initially by the generation of the cone pigment iodopsin, then by rhodopsin. The greater density of rods around the periphery of the retina make "averted" vision a useful method for threshold detection. Sensitivity to light can be impaired by vitamin A deficiency, or at high elevation, where oxygen depletion, or hypoxia, affects the retinal neurons. At high-elevation observatories, breathing oxygen will improve visual sensitivity (as well as other neural functions).

In spite of the eyes' high sensitivity, the "integration constant" is about $0.07 \mathrm{sec}$ onds, so it cannot match the faint detection capabilities of photographic emulsions or electronic detectors that can collect light over long exposures. 


\subsection{RESOLUTION}

As with any optical system, the eye is subject to resolution-limiting aberrations. Mental conditioning compensates for the effects of chromatic aberration, field curvature, and distortion, but with the large pupil size in dark conditions, spherical aberration becomes a small, but noticeable factor ( $20 \%$ over four orders of magnitude in brightness). Aberrations in the eyepiece or telescope are usually more important than those in the normal eye.

\subsection{CONTRAST THRESHOLD}

The visual contrast detection threshold is important in detecting a faint, diffuse coma against the sky background. This is not important in the limiting case of a faint and highly condensed comet (where the lack of a visible coma may prevent recognition of a comet), but in the case of a faint, extended coma, the apparent coma diameter may be dictated by the contrast detection threshold of the eye. Blackwell (1946) showed empirically that the visual contrast detection threshold changes by four orders of magnitude over the sensitivity range of the eye (more like one to two orders of magnitude over the scotopic range of the eye) and is also a function of the angular size of the target.

\subsection{PERCEPTION AND EXPERIENCE}

Seeing and recognizing faint and small objects is improved through practice and experience. The better observers are generally those who have spent much time looking through telescopes and have become familiar with the particular optical characteristics of the telescope. Training allows the observer to recognize faint or low-contrast features that the average person might have difficulty seeing. Well-known visual observers, such as E.E. Barnard, had a very highly developed sense of visual perception with the telescope. Today, few professional astronomers spend any time looking through an eyepiece, while many amateurs have highly developed skills for detecting faint and/or tiny objects through the telescope. Unfortunately, there will always be a few visual amateur astronomers who may be influenced by peer pressure or visions of fame to see something they think they should be able to see or very much want to see. Evaluating marginal observations may be very difficult, even when the observer attempts to be as objective as possible.

Additional information on the characteristics of vision can be found in reviews such as Fry (1965) and Williams and Becklund (1972).

\section{Visual Comet Discoveries}

Today, visual comet discoveries account for roughly one-fourth of all comet discoveries. Statistics of comet discoveries (Everhart, 1967; Kresak, 1982; Rudenko, 1986) show that most visual discoveries occur when comets are at $\mathrm{r}<2 \mathrm{AU}$ and $\Delta<1.5 \mathrm{AU}$ ( $r=$ the heliocentric distance and $\Delta=$ the geocentric distance). This geometric selection effect was well-shown by Kresak (1982), who plotted the loci of constant difference between apparent and absolute magnitude plus the effects of background illumination and extinction. This plot shows a peak envelope depth at about $35^{\circ}$ solar elongation for total magnitude $\mathbf{M}<10$. The peak discovery density in terms of solar elongation is shown by 
Rudenko (1988) to occur at about $40^{\circ}$, falling to half at $75^{\circ}$, and half again at $105^{\circ}$. The high rate of cometary brightening within $1 \mathrm{AU}$ of the Sun favors frequent visual searches within solar elongations $<90^{\circ}$. (However, since the chances of visual discovery are better within $100^{\circ}$ of the Sun, more time is spent in that area, and so the visual discovery statistics become skewed.) Kresak (1982) further reviews the observational selection effects for both visual and photographic discoveries. Selection effects are particularly important in estimating the comet population distribution.

Visual searches have two potential advantages over equivalent photographic ones: relatively high scanning speed, and rapid feedback and response to suspected comets. The trained eye, scanning through a wide-field eyepiece, can cover an area of some 400 square degrees in an hour. Assuming a magnification that matches the dark-adapted pupil, the telescope aperture effectively determines the eyepiece field. As Blackwell (1946) showed, the eye's contrast detection threshold is better for larger targets superimposed on a background. Thus, fainter diffuse comets can be detected above the sky background at higher magnification (i.e., aperture, for a given exit pupil). With a $0.4-\mathrm{m}$ telescope, the threshold for extended objects is about total magnitude 12. With smaller apertures and correspondingly larger fields, the time to scan a given area is shorter, but with a correspondingly brighter limit. Therefore, the single visual observer must balance aperture against the area and frequency to be scanned. The visual detection threshold may be improved by using both eyes through binoculars or a double telescope system, but binocular eyepieces sharing the same input beam with beamsplitters tend to have inferior throughput. The other advantage of immediate feedback is help in identifying motion and alerting other observers for confirmation of the discovery and acquisition of astrometric positions.

There are several schemes for comet sweeping. The one chosen is usually dictated by mechanical constraints of the telescope or by the observer's preference. Some observers build up a raster scan of barely overlapping fields sweeping in right ascension and declination, or azimuth and elevation, while a few others systematically search arbitrarily within defined boundaries, hoping to see a comet passing through the field. Although the latter method allows more careful inspection of a smaller area, a statistical study has not been made comparing the relative success of the methods.

The primary disadvantage of visual surveys is the bright magnitude limit as compared with that of photographic surveys. Photographic discoveries often go down to about magnitude 16, while visual discoveries are rarely fainter than magnitude 10. Another disadvantage is that the observer must either become extremely familiar with the locations of background nebulae or frequently consult maps and charts to initially identify a new comet. Determining the location of a comet suspect may be quite difficult, especially if the sky is brightening and reference stars become invisible, or if the object is setting behind a landscape feature. Obtaining a position with reasonable precision from the initial observation is critical for confirmation and follow-up. A worthwhile aid might be a camera with a fast lens, always mounted and bore-sighted with the telescope, to take a short (even unguided) exposure of the suspect. The photograph need not have a strong image of the suspected comet, if the location of the eyepiece center is known relative to brighter stars recorded in the field.

It is difficult to determine how efficient visual comet searches are. Because photographic discoveries are usually made when the comet is fainter, there is no way of knowing how many brighter comets are missed, and there are few statistics on unsuccessful searches. Statistical studies of visual comet discoveries (Everhart, 1967; Kresak, 1982; Machholz, 1985; Rudenko, unpublished) show an asymmetry favoring morning discoveries. This is most apparent for elongations of $35^{\circ}$ to $60^{\circ}$ and is caused by the orbital motion 
of the Earth modifying the geocentric velocity vector distribution in a way much like the diurnal variation of meteor rates (Kresak, 1982). In any case, it is apparent that the most successful visual discoverers are the ones who persist in searching and build up experience in recognizing threshold suspects.

\section{Brightness Estimates}

After astrometry, total visual magnitude estimates comprise the largest body of data available on comets. Largely supplanted today by charge-coupled device (CCD) photometry through spectrally selective filters and precisely defined apertures, from which production rates of several species and dust can be determined, visual magnitude estimates may still be useful in monitoring cometary activity as a function of heliocentric distance and studying secular variations over many orbits. A total visual magnitude estimate usually refers to the integrated brightness of the head (Green and Morris, 1987), but in one discussion (Marsden and Roemer, 1982), it is the integrated brightness of both the head and the tail. In cases of small phase angles, there might be an unknown contribution from a well-developed tail in the line-of-sight of the head, but this is not common.

Estimating a comet's brightness is not as simple as it may seem. The brightness profile of the extended image of a comet can vary from an almost uniform blob to a very condensed, almost starlike form. A technique for comparing the total brightness of an extended comet with point-like stars of known brightness was developed by Bobrovnikoff (1941). A comparison star and the comet are racked out of focus, until they have a similar size at the same focus setting, in an attempt to compare their surface brightnesses. Unfortunately, this means that the comet is also out of focus, but at least both can be quickly compared. Sidgwick (1955) introduced the method of placing the star out of focus until its surface brightness matches from memory the average in-focus comet surface brightness. This allows brightness estimates to be made of fainter comets that would be invisible when out of focus. Beyer (1952) used a method of comparing the extinction of grossly out-offocus images of the comet and star against the sky background. Morris (1979) introduced a method of placing the star and comet out of focus by different amounts to better normalize their appearance. Each of these methods suffers from systematic errors of varying degrees due to the character of the comet, the observing conditions (mostly sky brightness), and the optics used (Roemer, 1976; Meisel and Morris, 1976 and 1982).

There is considerable debate on quantifying the effects of observer experience, aperture, observing methods, degree of condensation, and observing conditions. The recent well-observed apparitions of comets P/Giacobini-Zinner and P/Halley provide wellsampled examples of the capabilities of visual magnitude estimates (Edberg and Morris, 1986; Bouma, 1987; Edberg, 1988). Edberg (unpublished) has analyzed some 1,000 raw observations of $\mathrm{P} /$ Giacobini-Zinner archived by the International Halley Watch and found that there was typically a two-magnitude range in reported brightness (Fig. 2). Analysis of the data showed that (1) more experienced observers report brighter values and have less scatter, (2) aperture correction did not reduce the scatter, but did introduce a slight zero offset, and (3) there was very large scatter in estimating the coma diameter and degree of condensation. One could conclude that the discrepancy in coma diameter may reflect varying sky conditions and/or optical configurations that would also affect the magnitude estimates. A similar analysis is being conducted on the much larger $\mathrm{P} / \mathrm{Halley}$ estimates, but a preliminary report using only eight days (Edberg and Morris, 1986) also shows that the scatter 


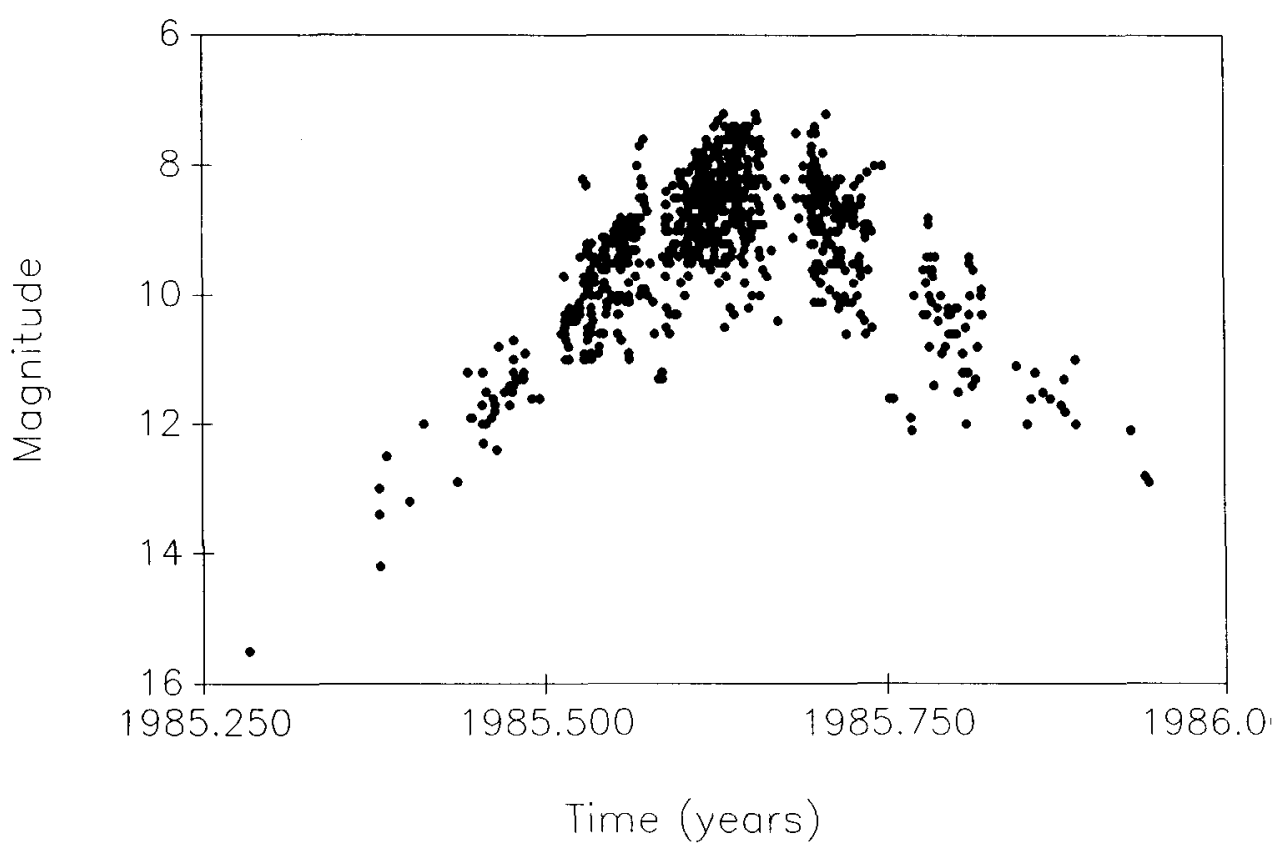

Fig. 2. Over 1,000 estimates of the total visual magnitude of $\mathrm{P} /$ Giacobini-Zinner during nine months in 1985, as compiled by S. Edberg as part of the International Halley Watch.

was a function (in order of importance) of experience, coma morphology, and instrumentation.

Uses of visual total magnitude estimates include prediction of future brightness and activity level when no other data are available. Secular changes in these cometary parameters can be searched for as well. Newburn and others (Newburn, 1979, 1981; Newburn and Yeomans, 1982; Divine et al., 1986) made extensive use of the visual magnitude estimates of P/Halley to estimate production rates and spacecraft flyby environments for 1986. Such efforts are difficult for brightness estimates made prior to the 1950s or 1960s, which require a careful study of the actual observational records to understand exactly how they relate to contemporary estimates (see, for example, Marcus, 1986).

The study of secular changes in cometary brightness is fraught with difficulty, again because of the calibration required between recent and past observations. The interpretation of the data and their meaning is open to considerable question. Hughes (1983) is an example of this work.

The standard magnitude equation (Green and Morris, 1987):

$$
\mathrm{m}_{1}=\mathrm{H}_{\mathrm{O}}+5 \log \text { delta }+2.5 \mathrm{n} \log \mathrm{r}
$$

is often used to describe a comet's brightness behavior. Here, $\mathrm{H}_{\mathrm{O}}$ is the absolute magnitude (defined at delta $=r=1$ ), delta is the comet's distance from Earth, $r$ is the comet's distance from the Sun, and $n$ is the exponent by which brightness varies inversely with $r$. 


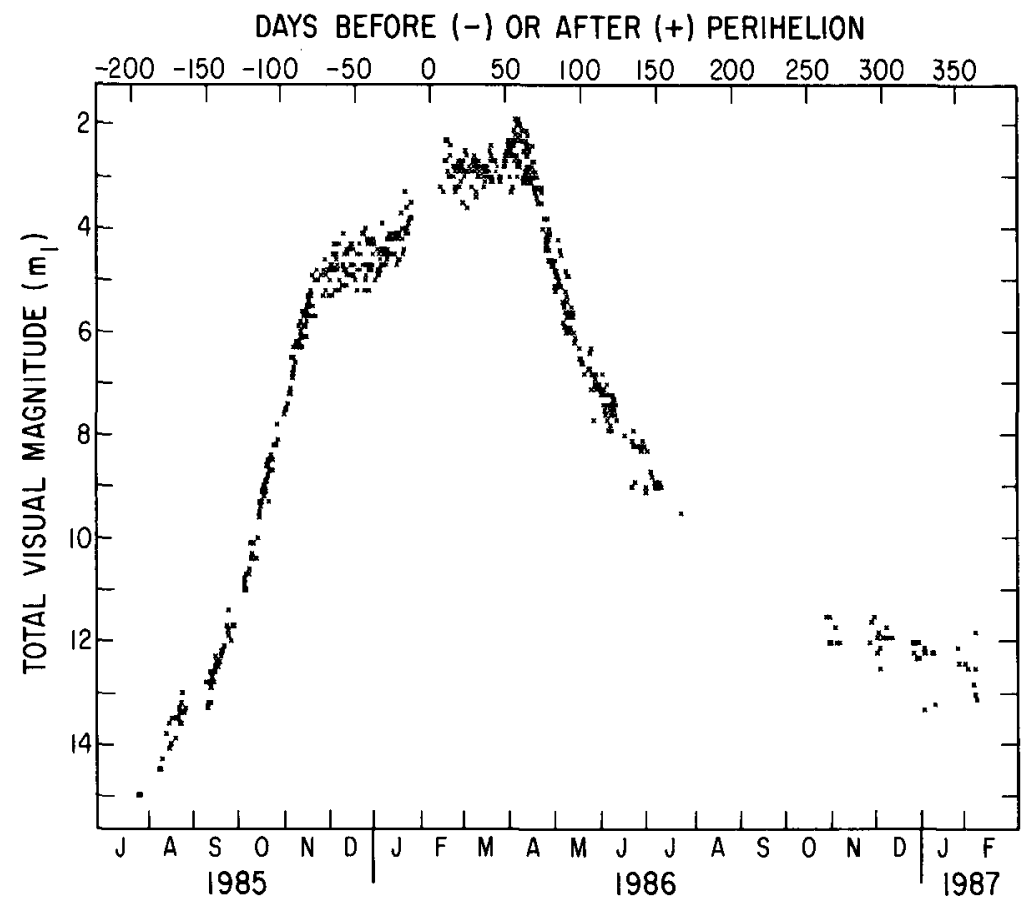

Fig. 3. The total visual magnitude of $\mathrm{P} / \mathrm{Halley}$, adapted from Green and Morris (1987), showing the scatter from a statistically significant sample.

While such an equation is convenient for initial projections of a newly discovered comet's brightness, it may not accurately represent the actual light curve. Green and Morris (1987) found, for example, that $\mathrm{P} / \mathrm{Halley}$ 's brightness was better represented with several segments of the orbit having different values of $\mathrm{H}_{\mathrm{O}}$ and $\mathrm{n}$.

One potential advantage of visual brightness estimates is that there are many observers well-distributed in longitude and, in principle, more likely to identify rapid changes of activity. Such work can be particularly useful in alerting others to unusual activity. However, changes in brightness due to increased short-term dust production, such as jets becoming active as they rotate into sunlight, may not appear to change the total magnitude of a comet significantly, because the jets may constitute a small fraction of the total light of the coma, and dust jets may take days to dissipate. Comet Halley's 7.4-day periodicity in brightness is much more easily seen using small diaphragms close to the central condensation than in total brightness integrated over the whole coma. This fact, coupled with the intrinsic scatter of visual estimates, makes it very difficult to identify the 7.4-day periodicity in the visual light curve (Fig. 3). With less active comets, such as P/SchwassmannWachmann 1, eruptive episodes may occur infrequently enough so that they have a substantial effect on the total brightness. In fact, "outbursts" of this comet have often been reported first by visual observers. 
A major disadvantage of visual magnitude estimates is that even for selected, experienced observers, they have a typical scatter of \pm 0.4 magnitudes (Green and Morris, 1987). The most important problem in using visual total magnitude estimates is that they refer to unknown relative contributions of continuum and $\mathrm{C}_{2}$ emission and are difficult, if not impossible, to interpret in quantitative physical terms (Fischer and Huttemeister, 1987).

\section{Drawings}

Since the eye can detect low-contrast and small features in the telescope, it has the potential for recording changes in coma morphology that can be used to make inferences about the rotation state of the nucleus (Sekanina, 1989). Unfortunately, most visual observers today have not developed the drawing skills or micrometer techniques to accurately portray what they see through the eyepiece. Visual drawings are more likely to be used by someone other than the observer, so it is important that they be interpretable. Recent drawings made of Comet Halley provide a good opportunity to evaluate their usefulness in the context of past apparitions and in comparison with recent CCD imagery. In general, the drawings do not fare well. The principal problem is that rarely do observers use aids such as filar micrometers or eyepiece reticules to measure position angles and sizes of features. For observers of past apparitions of Comet Halley, such techniques were common practice.

The other problem is one of interpreting different drawing "styles." These styles range from fairly realistic to very abstract and schematic. Almost all observers will tend to exaggerate the contrast of local features, while ignoring the larger, lower frequency gradients in the coma. Such "spatial filtering," although photometrically inaccurate, is an advantage of visual observing. Enhancement of digital CCD images to show coma features attempts to do the same thing. Figures 4 and 5 illustrate variations in drawing styles in 1910 and 1985.

Current investigators have found some drawings to be useful in finding nucleus rotation solutions constrained by the dust emission pattern. One good example is Sekanina's study of P/Swift-Tuttle (Sekanina, 1981), where totally independent drawings by Bond and Winnecke displayed a high degree of consistency, which suggests that they could be used with a relatively high degree of confidence. This is not always the case, as one can see by comparing drawings of P/Halley in 1910 by Innes, Worssell, and Ricco at about the same time on May 21, 1910 (Fig. 4). Sekanina has used Baldet's drawings of $\mathrm{P} /$ Pons-Winnecke and P/Schwassmann-Wachmann 3 in studying the fan-shaped comae when those comets were close to the Earth (Sekanina, 1989). There may be cases where position angles and extents of features may be more useful than a drawing, but the description must be complete and unambiguous to be useful quantitatively.

\section{Future Role of Visual Observations of Comets}

\subsection{VISUAL DISCOVERIES}

Visual searches will continue to play a role in finding comets, especially those that brighten rapidly near the Sun. Although photographic or electronic comet searches are possible in principle, in practice they do not cover the whole sky every day or employ the army of people necessary to extract and follow up possible comets. The efficiency with 


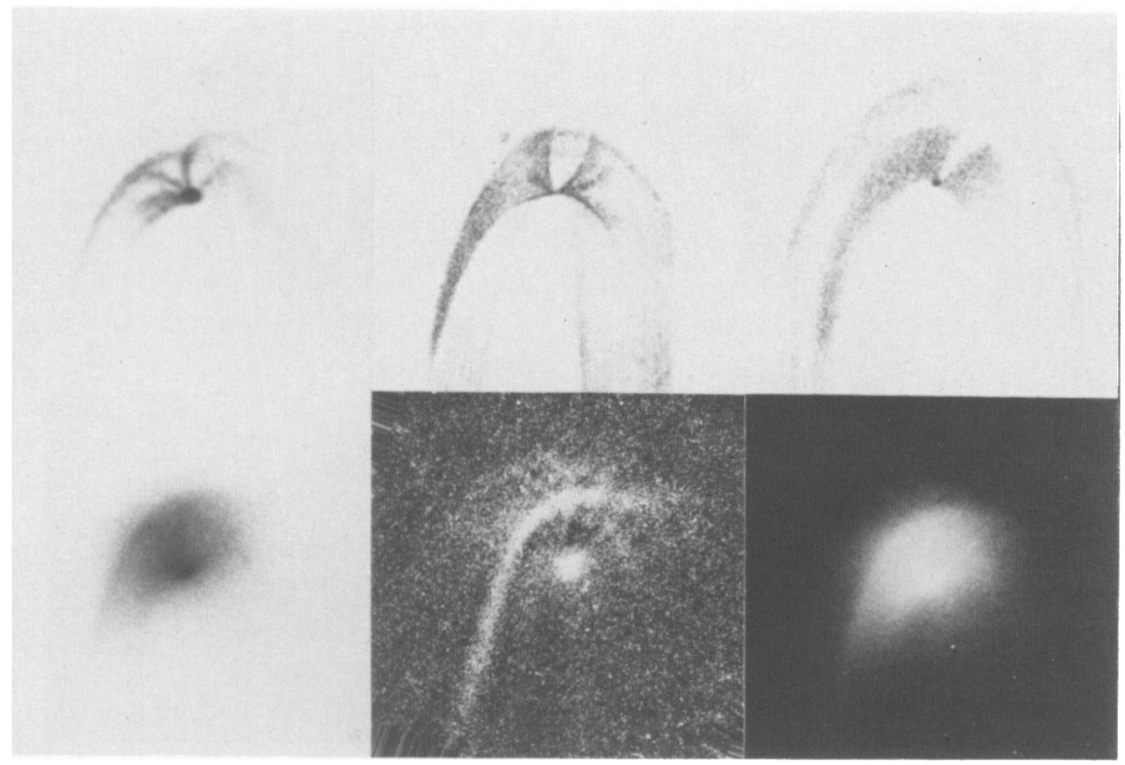

Fig. 4. Examples of styles in drawings of P/Halley by (left to right) A. Ricco, R. Innes, and $\mathrm{W}$. Worssell compared with straight negative, edge enhanced, and straight positive photographs by C. Lampland at the Lowell Observatory on 1910 May 21.3. The scale and orientation (Sun up) are approximately normalized.

which the trained visual observer with good weather can search large areas and rapidly verify suspected comets guarantees that a significant fraction of new discoveries will be made visually. It should be noted, however, that systematic photographic surveys, such as that of the Shoemakers at Palomar, have reduced the percentage of visual discoveries from about $50 \%$ to $25 \%$ in the last ten years. The efficiency of the Infrared Astronomical Spacecraft (IRAS) in discovering comets in 1983 has also had an effect on discovery statistics. Future plans for ground-based photographic and spaceborne infrared surveys would undoubtedly have a significant impact on the success of visual searches, but, with the current funding limitations for such projects, visual searches will remain a vital activity.

\subsection{VISUAL MAGNITUDE ESTIMATES}

Visual total magnitude estimates will continue to be made, because they can be compared with old observations and because they can be made easily by a large number of observers. However, as electronic detectors and filters become less expensive and more readily available, an increasing amount of high-precision photometric data will be obtained. The application of inexpensive CCD systems and plate scales appropriate to show the whole comet will provide more precise total magnitudes of comets, and the use of filters will permit more precise monitoring of gas and dust production.

For visual observations, improvements can be made now by establishing a better standard for magnifications and/or apertures used. The estimates and application of the degree of condensation might be investigated to understand why there is such a disparity be- 


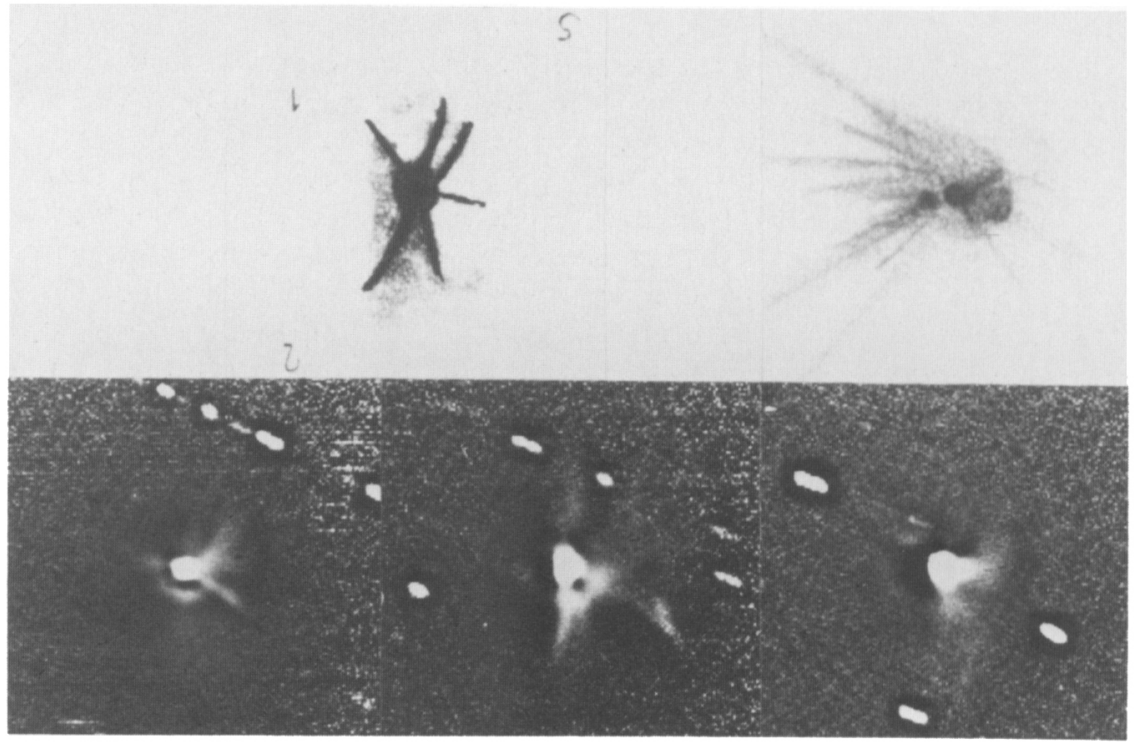

Fig. 5. Examples of drawings of P/Halley by A. Peres with the $0.8-\mathrm{m}$ Meudon observatory refractor on $1986 \mathrm{Dec}$. $15.9 \mathrm{UT}$ (left) and S. O'Meara with the 0.2-m Harvard College Observatory refractor (right) and enhanced CCD images taken with the $1.5-\mathrm{m}$ Catalina $\mathrm{Ob}$ servatory reflector by S. Larson and D. Levy on December 15.2, 16.2, and 17.2, 1985. The scale and orientation (north up, east to the left) is approximately normalized.

tween observers. The large number of observers may still provide useful identification of short-term increases of brightness (outbursts). The editors of and contributors to the International Comet Quarterly and its database of magnitudes will continue to characterize the gross brightness behavior of many comets and serve as a source of confirmation of unusual activity aiding the interpretation of other data sets.

\subsection{DRAWINGS}

As in the past, historical visual drawings will be used in critical situations when there is no other information. For the most part, however, modern drawings do not provide the information required for quantitative analysis. This may be because observers today rarely use their drawings for any detailed study themselves, and therefore they cannot appreciate the need to go through the additional trouble of, for example, making position angle and distance measurements. The proliferation of larger aperture telescopes on precision mountings and good area detectors, from hypersensitized photographic emulsions to CCD cameras, makes it possible to obtain systematic records of coma and tail morphologies that are suited for quantitative analysis with relatively little effort. With ever increasing numbers of competent astrophotographers available, most visual drawings may become products of recreational astronomy. 


\subsection{A FURTHER NOTE}

Although outside the intended scope of this paper, we note that the role of future visual observations as contributions to cometary research will depend upon how well the observer understands and executes the needs of the scientific method. In spite of efforts to channel amateur resources in the IHW (Edberg, 1983), Edberg noted that "many experienced amateur observers had difficulty in maintaining an unbiased, scientific attitude about their results and their methods in obtaining them.... The amateur community, as a group, does not have a good understanding of the scientific method...." (Edberg, 1988). This result of the IHW's experience with amateur astronomers may be a manifestation of the current deemphasis of serious science education, a lack of professional involvement in fostering professional/amateur coordination, unreasonable expectations from a hobby, or some combination of these. It is clear, though, that with a serious effort, amateurs can make useful and important contributions.

The many serious amateurs eager to make contributions to cometary research constitute a sizable resource whose potential is only partly realized. The work of a few of the leading amateurs can serve as a model to encourage the thoughtful acquisition of data and the study of cometary phenomena by others with telescopes at good sites.

\section{Acknowledgements}

We wish to thank R. Bouma, P. Collins, D. Fischer, D. Green, J. Marcus, B. Marsden, C. Morris, J. Scotti, and Z. Sekanina for helpful discussions, R. Newburn and an anonymous reviewer for suggestions improving the manuscript, and $\mathrm{A}$. Peres and $\mathrm{S}$. O'Meara for their contributions to the IHW archive that are used here.

\section{References}

Beyer, M. (1952) 'La Physique des Cometes,' Mem. Soc. Roy. de Liege 13, 236. Blackwell, H.R. (1946) 'Contrast thresholds of the human eye,' J. Opt. Soc. Am. 36, 624.

Bobrovnikoff, N.T. (1941) 'Investigations of the brightness of comets, Part 1 and 2,' Contributions from the Perkins Observatory, Nos. 15 and 16.

Bouma, R.J. (1987) 'The lightcurve of P/Halley: An analysis of amateur observations,' Proc. International Halley Seminar, Alden Biesen, Belgium.

Divine, N., Fechtig, H., Gombosi, T.I., Hanner, M.S., Keller, H.U., Larson, S.M., Mendis, D.A., Newburn, R.L., Reinhard, R., Sekanina, Z., and Yeomans, D.K. (1986) 'The Comet Halley dust and gas environment,' Space Science Reviews 43, $1-104$.

Donn, B., Rahe, J., and Brandt, J.C. (1986) 'Atlas of Comet Halley 1910 II,' NASA SP-488, Washington, D.C.

Edberg, S. (1983) 'International Halley Watch amateur observer's manual for scientific comet studies,' JPL Publication 83-16, Pasadena, California.

Edberg, S. (1988) 'Working with amateur astronomers: Lessons from the International Halley Watch,' in Stargazers-Amateur Contributions to Astronomy (Proc. IAU Colloquium 98), S. Dunlap and M. Gerbaldis (eds.), Springer-Verlag, Berlin, pp. 95-99. 
Edberg, S., and Morris, C.S. (1986) 'Observational factors affecting studies of P/Halley's visual light curve,' in Proc. 20th ESLAB Symposium on the Exploration of Halley's Comet, ESA SP-250, Vol. I, Heidelberg, pp. 609-612.

Everhart, E. (1967) 'Comet discoveries and observation selection,' Astron. J. 72, 716726.

Fischer, D., and Huttemeister, S. (1987) 'Comet P/Halley: Visual magnitude estimates and gas production,' in Symposium on the Diversity and Similarity of Comets, ESA SP-278, pp. 599-605.

Fry, G.A. (1965) 'The eye and vision,' in Applied Optics and Optical Engineering, Vol. II, R. Kingslake (ed.), Academic Press, New York, pp. 1-76.

Green, D.W.E., Rokoske, T.L., and Morris, C.S. (1986) 'The ICQ photometric archive for P/Halley and other comets,' in Proc. 20th ESLAB Symposium on the Exploration of Halley's Comet, ESA SP-250, Vol. III, Heidelberg, pp. 249-251.

Green, D.W.E., and Morris, C.S. (1987) 'The visual brightness behavior of P/Halley during 1981-1987,' Astron. Astrophys. 187, 560-568.

Ho Peng Yoke (1962) 'Ancient and medieval observations of comets and novae in Chinese sources,' Vistas Astron. 5, 127-225.

Holetschek, J. (1896-1917) 'Untersuchungen uber die Grosse und Helligkeit der Kometen und Ihrer Schweife,' Denksch. Wiener Ak., Math. Naturwiss. K1. 63, $317 ; 77,503 ; 88,745 ; 93,201 ; 94,375$.

Hughes, D.W. (1983) 'Temporal variations of the absolute magnitude of Halley's comet,' Mon. Not. R. Astr. Soc. 204, 1291-1295.

Kresak, L. (1982) 'Comet discoveries, statistics and observational selection,' in Comets, L.L. Wilkening (ed.), University of Arizona Press, Tucson, pp. 56-82.

Kronk, G.W. (1984) 'Comets: A descriptive catalog,' Enslow, Hillside, NJ.

Machholz, D. (1985) 'A decade of comets: A study of 33 comets discovered by amateur astronomers between 1975 and 1984,' published by the author.

Marcus, J.N. (1986) 'Halley's true brightness in 1909-11 and comparison to 1985-86,' in Proc. 20th ESLAB Symposium on the Exploration of Halley's comet, ESA SP-250, Vol. III, Heidelberg, pp. 307-312.

Marsden, B.G. (1986) 'Catalog of cometary orbits,' International Astronomical Union, Cambridge, MA.

Marsden, B.G. and Roemer, E. (1982) 'Basic information and references,' in Comets, L.L. Wilkening (ed.), University of Arizona Press, Tucson, pp. 707-733.

Meisel, D.D., and Morris, C.S. (1976) 'Comet brightness parameters: Definition, determination and correlations,' in The Study of Comets, B. Donn, M. Mumma, W. Jackson, M.A. A'Hearn and R. Harrington (eds.), NASA SP-393, Washington, D.C., pp. 410-444.

Meisel, D.D., and Morris, C.S. (1982) 'Comet head photometry: Past, present and future,' in Comets, L.L. Wilkening (ed.), University of Arizona Press, Tucson, pp. 413-432.

Morris, C.S. (1979) 'A new method for estimating cometary brightness,' Comet News Service 79-1.

Morris, C.S. (1980) 'A review of visual comet observing techniques. I,' Internat. Comet Quart. 2, 69-73.

Morris, C.S., and Green, D.W.E. (1982) 'The light curve of periodic Comet Halley 1910 II,' Astron. J. 87, 918-923. 
Newburn, R.L. (1979) 'Physical models of Comet Halley based upon qualitative data from the 1910 apparition,' in The Comet Halley Micrometeoroid Hazard, ESA SP-153, ESTEC, Noordwijk, The Netherlands, pp. 35-50.

Newburn, R.L. (1981) 'A semi-empirical photometric theory of cometary gas and dust production: Application to Comet Halley's gas production rates,' in The Comet Halley Dust and Gas Environment, ESA SP-174, ESTEC, Noordwijk, The Netherlands, pp. 3-18.

Newburn R.L., and Yeomans, D.K. (1982) 'Halley's Comet,' Ann. Rev. Earth Planet. Sci. 10, 297-326.

Rahe, J., Donn, B., and Wurm, K. (1969) 'Atlas of cometary forms: Structures near the nucleus,' NASA SP-198, Washington D.C.

Roemer, E. (1976) 'Luminosity and astrometry of comets: A review,' in The Study of Comets, NASA SP-393, Washington, D.C., pp. 380-407.

Rudenko, M. (1986) 'Catalog of cometary discovery positions,' Internat. Comet Quart. 8, $117-129$.

Rudenko, M. (1988) 'Comet hunting by the numbers,' unpublished manuscript.

Sidgwick, J. (1955) 'Observational astronomy for amateurs,' Faber and Faber, London, p. 251.

Sekanina, Z. (1981) 'Distribution and activity of discrete emission areas on the nucleus of periodic Comet Swift-Tuttle,' Astron. J. 86, 1741-1773.

Sekanina, Z. (1989) 'Nuclei of two earth-grazing comets of fan-shaped appearance,' Astron. J. 98, 2322-2345.

Sekanina, Z. (1990) 'Cometary activity, discrete outgassing areas, and dust jet evolution,' this volume.

Williams, C.S., and Becklund, O.A. (1972) 'Optics: A short course for engineers and scientists,' Wiley-Interscience, New York.

Vsekhsvyatskii, S.K. (1958) 'Physical characteristics of comets,' 1964 translation, Israel program for scientific translations, Jerusalem, NASA TTF-80. 